\title{
Language, Theory of Mind and Autism Therapy
}

\section{Ackermann (Michel Ackermann)', H. Kyuchukov (Hristo Kyuchukov)²}

${ }^{1}$ Berliner Institut für Familientherapie, Berlin, DE

2 University of Silesia, Katowice, PL

\section{E-mail address:}

hkyuchukov@gmail.com

\section{Reprint address:}

Hristo Kyuchukov

University of Silesia in Katowice

Faculty of Ethnology and Educational Science

Bielska 62

43-400 Cieszyn

PL

Source: Clinical Social Work and Health Intervention Pages: $7-13$
Volume: 9

Issue: 2

\section{Reviewers:}

Michael Costello

University of Scranton School of education, USA

Roberto Cauda

University Catholica Clinica, Gemeli, Rome, IT

\section{Key words:}

Autism. Language. Theory of Mind. Therapy.

\section{Publisher:}

International Society of Applied Preventive Medicine i-gap

CSWHI 2018; 9(2): 7 - 13; DOI 10.22359/cswhi_9_2_01 @ 2018 Clinical Social Work and Health Intervention

\section{Abstract:}

This paper presents an overview of the literature regarding autism and role of language in the process of therapy with the autistic children and adults. The overview of the literature shows the importance of two factors: the use of syntactic structures and understanding of Theory of Mind tasks. The role of the language in the process of therapy of autistic children and adults is discussed. 


\section{Definition}

According to the International Classification of Diseases (ICD-10), autism is considered "a profound developmental disorder" (F84). A lifelong complex disorder of the central nervous system is the basis, especially in the area of perceptual processing, which begins in childhood. In the center is a serious relationship and communication disorder. The impact of the disorder hinders in a variety of ways the relationships with the environment, participation in life in the community, and the ability to integrate into society, as both cognitive, linguistic, motor, emotional, and interactional functions are affected. In addition, there are numerous behavioral problems, which can be particularly burdensome for the reference persons in everyday dealing with the autistic people.

A more generalized definition is provided by R. Davis (2012) who is an autistic person himself: "autism is essentially not an impairment of cognition or intellect, but of integration. The autist was not able to provide new information and experiences in his life and life like non-autistic or neurotypical persons"

Research on autism is ultimately also research about self-consciousness perceiving itself and drawing conclusions by perceptions: research on cognition. In the age of research on the neurophysiological implications of cognition, the research community here is perhaps on a threshold which could be compared with the transition from classical physics to quantum mechanics. We know that the old duality between mind and body is, in essence, a construction which is true-tolife because of our self-awareness, but which is in essence incorrect, just as classical physics functions according to Newton's laws, but only on the macro plane and not in the inner core. And just as in physics, the theory that connects both levels cannot yet be formulated conclusively. Until now there is no definite outline for a research on cognition bringing the observed brain-processes in a coherent relation to the communicative processes of the external world. Is this possible at all?

At least, it is probably true that consciousness exists on the systemic level through a complex interplay of perception, cognition and a communication between resulting (internal and external) processes, which arise when this interplay is imbued with emotions.

Language, in turn, has evolved in evolution as an additional element in the "space" between perception, cognition, communication and behavior. And autism as a neurological phenomenon in turn results from a lack of integration of the above-mentioned systemic "consciousness antagonists": Perception could not be integrated, or only partially, with internal processes (thoughts) and resulting behavior, which was co-guided by emotions. Our language as an integrative control instrument plays either as a very dominant (highly-functional Asperger's autism) or as a very slow (speech-impaired) tool for integration.

Current autism research is no coincidence for the reasons described above. It studies the role of consciousness and language (as a systemic partner of consciousness) in autism. The concept of 
consciousness in the autism area is further described by the "Theory of Mind".

The "Theory of Mind" (TOM), also called Naive Theory, describes in Psychology and other Cognitive Sciences the ability to make an assumption about the processes of consciousness in other persons and to recognize them in their own person as feelings, needs, ideas, intentions, expectations and opinions (Resch et al. 1999). One could also assume and formulate that consciousness needs, among other things, a process of 'mentalization' (another word for TOM) which arises by the fact that a self-conscious being achieves this state by perceiving similar processes of thought and feelings likewise in other equally conscious beings

- How mentalization is linked to language, and what role this plays in autism, is currently the most important field of research on autism and language. Here are some recent research results: It was assumed that on one hand, language skills play a fundamental role in the ability to TOM, but autism as a mental feature plays a greater role for the TOM than the language itself.

- In so-called "false belief" tests (false or missing assumptions about the internal condition of other persons), autistic children with speech disability and non-autistic children with a specific language disability were compared.

- The results confirmed that the autistic children had worse results in TOM than the non-autistic children. The tests were nonverbal to confirm this assumption (Colle, Baron-Cohen, Hill, 2007).
Furthermore, it was suggested that nonverbal TOM tests, compared to verbal TOM tests with highly functional autists, show that these would be worse in the non-linguistic tests than in the linguistic (as language for the TOM nevertheless plays an important role) (Lindsay et al, 2007 )

Causing for poorer nonverbal TOM results in high-functional (that is to say linguistically gifted) autists is perhaps also the fact that in autism the integration of visual and auditory signals is impaired. This means that mimic and gesture language cannot be "anticipated", or their perception cannot be supported by pictorial information and interpreted into other persons using TOM: The assumption or notion of what is happening in other people is much more difficult to implement, if not impossible, if there is a limited possibility of bringing together pictorial and auditory information (Silverman et al. 2010).

Regardless of possible implications for the TOM, this experiment initially only indicated that the integration of visual and auditory signals is important for a language understanding, which also anticipates the statement from the other by means of body language signals in order to make communication more fluid.

Despite language limitations, autists often have musical talents. This seems to be due to the fact that the corresponding brain areas (gyrus frontalis inferior) overlap for speech and music processing, but autists for as yet unknown reasons show stronger brain activity as control persons, whereas speech shows weaker activity (Lai et al. 2012). 
Moreover, by means of mere tones or noises, the condition of another being can be "read" much more easily and more directly than by the addition of verbal derivations: The difference between a menacing roar and a gentle purr can also be intuitively understood by children who are not yet mentally capable for TOM.

In turn, tonal languages such as e.g. Mandarin does not help Chinese autists to better cope with the problem of processing both emotional and semantic content.

The identified circumstances for autism and language are confirmed and supplemented by further research. It can be shown, for example, that the influence of syntactic structures (with the example of simple accessory sentences) exerts a direct influence on the possibility of mentalization (TOM).

Autistic children had significantly more difficulties with "false belief" tests, if they were exclusively about syntactically oriented language and their descriptions about communication and thinking ("I suspect my friend is just sad."). Less so, however, when it comes to directly related words of perception ("I see my sad friend."). Their ability to speak about a perceived state and at the same time to perceive others in this state appears to be particularly affected in the first case. When language names, as in the second case of wording, exclusively the perception of what is perceived ("My friend is sad"), the TOM succeeds better (Tager-Flusberg \& Joseph (2005); Lind \& Bowler (2009).
A systematic analysis of autistic children regarding age and speech ability at the time of diagnosis is also very revealing: children diagnosed earlier were significantly worse off after this field study than children who were later diagnosed with ASD (Salomone et al. 2016).

Equally interesting is a study which shows that autistic children are more oriented towards the non-semantically relevant sound components than linguistic-syntactic forms (Järvinen-Pasley et al. 2008). The priority of the processing is, however, also in autistic children on the syntactic structures, when language stimuli were used in competing linguistic versus sound (only perception-oriented) components. Obviously there are levels of perception processing in the autists, where what is perceived is not directly given to the cognition, but remains, as it were, in the mode of perception. Perception and cognition can be an indissoluble conflict, and it is difficult to decide whether a signal is meaningful, carries relevant information or is redundant, even if it is "appealing" on the sensory level.

Furthermore, another study shows that autists use less internal language than control groups. They translate inner images of perceived situations or things much more slowly into inner language than other people (Whitehouse 2006).

All these research findings suggest that there are distinct differences between the autistic and the non-autistic in speech processing in the brain, which on the one hand are related to, or influenced by, mentalization, but also due to the different processing of sound and 
content language components, which in turn also influence the TOM processes, as well as the limitations of autistic people, to synchronize visual and auditory signals well and to integrate them into perception and cognition.

\section{Language as therapeutic organ}

On one hand, language seems to be a complex regulative for social behavior and on other hand, it seems to be closely related to cognitive representations of human consciousness, whose material emergences it communicates reality via acoustical and visual signs. From this point of view, language remains functional and expands the communicative and cognitive behavioral repertoire of human beings. It may be considered too little as a system whose inherent dynamics have an reverse impact on the original inventor and in turn may cause him to further refine himself in collaboration with his partner organ: "linguistically cognition".

If we consider language to be more than a biological and systemic-cognitive organ in the described sense, it is also clear at this point why a language therapy for autistic people cannot be done as if language was a rational object similar to a tool with its own operating instructions and rules of use to which the autistic client has to conform and ultimately submit. Because every human being has his or her individual linguistic organ, just as every human being has an unmistakable fingerprint or an individual sexuality.

Language is in all probability not the result of a homogeneous neurological evolutionary process, but rather the systemic result of many specifically human developments, starting with the advancement of fine motor skills (tool making, movement rituals in the group) and the corresponding effects in syntactic and thus loudly oriented cognitive and communicative skills. This process includes mimetic developments, i. e. especially those of the ability to imitate sounds of the environment, furthermore the cooperative requirements of differentiated and situational communication, including the ability to deceive other beings or members of one's own group by phonetic significations. Emotional needs from a newly orientated parent-child communication also belong to this.

If we understand language in such a multifaceted system and background, it becomes clear that the autistic system calls for a correspondingly diverse and careful use of language:

The permanently intermixing use of completely different aspects of human language will cause severe confusion to autistic people, because in their neurological (speech) system the utmost challenge is to integrate aspects of speechbound communication much worse than "neurotypical" people. In a positive way (and in the sense of a >reframing `), this would mean that a specific autistic language could become highly specialized and learn with new situational modes of cognition, which, on the one hand, relieve them from a permanent total integration of the various language aspects. On the other hand, it would also offer the chance to develop and use language in its pure form on separate levels. (Jang et al., 2015) 
However, we still need an excursus in order to better understand the entanglement of language and consciousness. Even a systemically adapted speech design and therapy for autistic people should be able to embed its progresses in their self-awareness in a way that the playful re-evaluation of an altered speech experience can enter deeper layers of consciousness and not only allow for new linguistic tricks or just playing with spoken patterns.

\section{Conclusion}

Audio-sensory therapy makes it possible to experience an oriented position of one's own body and its perception in space and time. This requires a perception that can define its own position in the sense of a "center of perception" in order to position and orientate one's own body in relation to the outside world. For as human beings we are not just any biological material which is orientated "somehow" based on sensory information of the environment. Our inner and outer orientation in the environment is achieved through perceptual abilities that have enabled us in the course of evolution to navigate our own biological system in communicative-cognitive processes, the basis of which can be considered in the purposeful perception of our own organism, as a unit that can be modified and independently regulated in time and space. Only a person who experiences himself/herself as self-directed and adaptable in time can orientate himself/herself in the environment in a self-effective way. If such an ability has not been sufficiently developed, the basics for an effective control of one's own life are missing.
Wirth regard to the area of autism and speech, our intended therapeutic intervention would be based on the assumption that the work with >Noit< has stabilized the perception of the internal and external borders for the clients to such an extent that a changed work with linguistic interventions in the therapeutic work would be possible. We would aim at testing a therapeutically varied language in terms of its sensory-communicative effectiveness, which is based on the speech bodies themselves (onomatopoetic parts of the words bodies) on the one hand, and on the other hand increases the flexibility of the (often compulsively rigid) use of words through playful access to semantic parts.

Language, for its part, should function as a vocally and noisy stimulating self-orientation, initially still independent of an intended communication use of language. We would assume that the therapeutic experience of a varied language as an additional (besides of a Noit) orienting sound tool can bring about a change in the communicative orientation of our autistic clients.

\section{References}

1. COLLE, L., BARON-COHEN, S. \& HILL, J. (2007). Do Children with Autism have a Theory of Mind? A Non-verbal Test of Autism vs. Specific Language Impairment. Journal of Autism and Developmental Disorders, 37, p. 716-723

2. DAVIS R (2012) Autism and the Seeds of Change: Achieving Full Participation in Life through the Davis Autism Approach.

3. ICD-10 (1994) Classification of Mental and Behavioural Disorders. Chirchill Livingstone. 
4. ARVINEN-PASLEY A (2007) Is the linguistic content of speech less salient than its perceptual features in autism? Journal of Autism and Development Disorders, 38(2):239-48.

5. JIANG J ET AL. (2015) Perception of Melodic Contour and Intonation in Autism Spectrum Disorder: evidence from Mandarin speakers. Journal of Autism and Developmental Disorders, Vol. 45, (7) pp 20672075.

6. LAI G ET AL. (2012) Neural systems for speech and song in autism. Neurology, Brain, 135, (3): 961-975.

7. LIND SE, BOWLER DM (2009) Language and theory of mind in autism spectrum disorder: the relationship between complement syntax and false belief task performance. Journal of Autism and Developmental Disorders 39, (6): 929-937.

8. LINDSAY, G., DOCKRELL, J., \& STRAND, S. (2007). Longitudinal patterns of behaviour problems in children with specific speech and language difficulties: Child and contextual factors. British Journal of Educational Psychology, 77, 811-828.

9. RESCH F ET AL. (1999) Developmental Psychopathology of Childhood and Adolescence. An Instructional Book Belz: Weinheim.

10. SALOMONE E ET AL. (2016) Child's verbal ability and gender are associated with age at diagnosis in a sample of young children with ASD in Europe. Child Care and Health Development, 42, (1): 141-145.

11. SILVERMAN L ET AL. (2010) Speechand-gesture integration in high functioning autism. Cognition, 115 (3): 380-393.

12. TAGER-FLUSBERG \& JOSEPH (2005) Why language matters for theory of mind (pp. 298-318). In: Astington, \& Baird (Eds.), New York, NY, US: Oxford University Press, Whitehouse WHITEHOUSE AJ (2006) Inner speech impairments in autism. Journal of Child Psychology and Psychiatry, 47, (8): 857-865. 